\title{
Is the July Effect Real in Patients Undergoing Endoscopic Retrograde Cholangiopancreatography?
}

\author{
Tae Yoon Lee ${ }^{1}$ and Yousuke Nakai ${ }^{2}$ \\ ${ }^{1}$ Division of Gastroenterology, Department of Internal Medicine, Konkuk University School of Medicine, Seoul, Korea, ${ }^{2}$ Department of \\ Gastroenterology, Graduate School of Medicine, The University of Tokyo, Tokyo, Japan
}

See "A Nationwide Assessment of the "July Effect" and Predictors of Post-Endoscopic Retrograde Cholangiopancreatography Sepsis at Urban Teaching Hospitals in the United States" by Rupak Desai, Upenkumar Patel, Shreyans Doshi, et al., on page 486-496.

We would like to comment on the first study investigating the "July effect" in post-endoscopic retrograde cholangiopancreatography (ERCP) sepsis at teaching hospitals in the United States. ${ }^{1}$ This study is particularly significant because the July effect has not been previously evaluated in relation to the incidence of post-ERCP sepsis. Hypothetically, post-ERCP complications will be higher in the early academic year, when trainees are relatively inexperienced in the ERCP procedure and in pre- and post-ERCP patient care. It is logical to assume that the undeveloped skill set of newcomers in the early academic months will lead to worse outcomes as compared with those in the remainder of the academic year, and this phenomenon is referred to as the "July effect". However, there is no convincing evidence to demonstrate the presence of the July effect in post-ERCP complications.

The National Inpatient Sample (NIS) database in the United States provides large and reliable data that can be used to evaluate whether a July effect truly exists. In this study, the authors utilized the NIS database to compare the incidence of post-ERCP sepsis in the early (July to September) and late

Received: July 3, 2019 Accepted: July 10, 2019

Correspondence: Yousuke Nakai

Department of Gastroenterology, Graduate School of Medicine, The University of Tokyo, 7-3-1 Hongo Bunkyo-ku, Tokyo 113-8655, Japan

Tel: +81-3-3815-5411, Fax: +81-3-5800-9801, E-mail: ynakai-tky@umin.ac.jp

ORCID: https://orcid.org/0000-0001-7411-1385

cc This is an Open Access article distributed under the terms of the Creative Commons Attribution Non-Commercial License (http://creativecommons.org/ licenses/by-nc/3.0) which permits unrestricted non-commercial use, distribution, and reproduction in any medium, provided the original work is properly cited.
(October to June) academic months in 481,193 ERCP procedures between 2010 and 2014. A higher incidence (9.4\% vs. $8.8 \%, p<0.001$ ) and higher odds (odds ratio, 1.07) of post-ERCP sepsis were observed in ERCP procedures performed during the early academic months. However, the in-hospital mortality rate ( $7 \%$ vs. $7.5 \%, p=0.072$ ), length of hospital stay, and total hospital charges in patients with post-ERCP sepsis were equivalent between the 2 time points. ${ }^{1}$

Although no data are flawless in these types of analyses, it is impressive to know that, as far as this study can inform, there is marginal but statistically significant evidence suggesting that a July effect exists in the occurrence of post-ERCP sepsis. This study raises the question of why it is important to know the effect of trainees on post-ERCP complications. Although the results have been rather variable among studies, several studies have reported reduced efficiency in health-care delivery at the beginning of the academic year among numerous medical conditions, as demonstrated by increased length of hospital stay and mortality. ${ }^{2}$

Contrary to the results of this study, another study using the NIS database reported that a July effect does not exist in the occurrence of post-ERCP complications. It also found that the rates of post-ERCP pancreatitis did not differ in teaching hospitals throughout the academic year, suggesting that the presence of in-training fellows and residents is not a risk factor for the development of post-ERCP pancreatitis. ${ }^{3}$ However, Cheng et al. revealed that ERCP procedures that involved trainees had a higher risk for post-ERCP pancreatitis. ${ }^{4}$

It is unclear why the results of studies investigating the July 
effect in post-ERCP complications were inconsistent. Both the involvement of inexperienced trainees in ERCP procedures and inadequate periprocedural care can pose a risk of higher post-ERCP complications. "Lack of technical competence", "lack of supervision", and "handoff errors" were the common causative factors of post-ERCP complications in settings with trainees. A longer procedural time, additional unskilled maneuverers, and lack of knowledge of post-ERCP care (e.g., antibiotic use) could have led to the higher incidence of post-ER$\mathrm{CP}$ complications. Close supervision by attending physicians is important because it can mitigate the potential risks caused by novice residents and endoscopy fellows. Handoff errors may be decreased by implementing a well-structured handoff system.

This study is a good start in evaluating whether novice trainees have a negative effect on post-ERCP outcomes. Whether post-ERCP sepsis is related to an unskilled procedural technique or to the overall periprocedural care is still controversial. The observation of a high incidence of post-ER$\mathrm{CP}$ sepsis suggests that close supervision and strict mentorship by attending endoscopists are warranted at academic centers in light of reports of hourly increasing mortality of not properly treated sepsis. Although the NIS is a large and reliable database, we wonder if it collects the type of data needed to determine if there is a difference in post-ERCP sepsis related to inexperience. For example, the authors could use only In- ternational Classification of Diseases- 9 codes in the absence of laboratory values and procedure details that could have made the definition of post-ERCP complications more accurate. Accordingly, there should be continued efforts to investigate post-ERCP complications that can be anticipated to occur with inexperience or with trainee involvement. This will provide the safest possible care within the context of training new residents and endoscopy fellows.

Conflicts of Interest

The authors have no financial conflicts of interest.

\section{REFERENCES}

1. Desai R, Patel U, Doshi S, et al. A nationwide assessment of the "July effect" and predictors of post-endoscopic retrograde cholangiopancreatography sepsis at urban teaching hospitals in the United States. Clin Endosc 2019;52:486-496.

2. Barry WA, Rosenthal GE. Is there a July phenomenon? The effect of July admission on intensive care mortality and length of stay in teaching hospitals. J Gen Intern Med 2003;18:639-645.

3. Schulman AR, Abougergi MS, Thompson CC. Assessment of the July effect in post-endoscopic retrograde cholangiopancreatography pancreatitis: Nationwide Inpatient Sample. World J Gastrointest Endosc 2017;9:296-303

4. Cheng CL, Sherman S, Watkins JL, et al. Risk factors for post-ERCP pancreatitis: a prospective multicenter study. Am J Gastroenterol 2006;101:139-147. 